\title{
General Psychiatry Neurocysticercosis presenting as bipolar disorder: a case report
}

\author{
Surbhi Batra (D , ${ }^{1}$ Sumit Kumar, ${ }^{2}$ Lokesh Singh Shekhawat ${ }^{1}$
}

To cite: Batra S,

Kumar S, Shekhawat LS. Neurocysticercosis presenting as bipolar disorder: a case report. General Psychiatry 2021;34:e100663. doi:10.1136/ gpsych-2021-100663

Received 15 September 2021 Accepted 16 November 2021

Check for updates

(C) Author(s) (or their employer(s)) 2021. Re-use permitted under CC BY-NC. No commercial re-use. See rights and permissions. Published by BMJ.

${ }^{1}$ Department of Psychiatry, Atal Bihari Vajpayee Institute of Medical Sciences and Dr Ram Manohar Lohia Hospital, New Delhi, Delhi, India

2Department of Radiology, Atal Bihari Vajpayee Institute of Medical Sciences and Dr Ram Manohar Lohia Hospital, New Delhi, Delhi, India

Correspondence to Dr Lokesh Singh Shekhawat; drlokesh4@gmail.com

\begin{abstract}
Neurocysticercosis is the most common neuroparasitosis caused by the larval stage of Taenia solium. The most common manifestations include seizures and hydrocephalus. Psychiatric abnormalities are relatively rare but depressive symptoms are frequent in patients with neurocysticercosis. However, mania as a presentation is relatively rare. Pregnancy and the postpartum period are relatively vulnerable times and they can lead to reactivation of existing neurocysterci lesions. We are discussing the case of a 23-year-old female patient with neurocysticercosis leading to the reactivation of lesions in the peripartum and postpartum period leading to bipolar affective disorder. Improvement in the patient was seen with a combination of antipsychotics, antihelmintics, antiepileptics and steroids, along with improved radiological signs of neurocysterci lesions. Although neurocysticercosis is a common illness, its prevalence presenting as a manic episode is merely $2.6 \%$ and, hence, missed easily. Therefore, it is important to rule out organic aetiology in patients even with a classic presentation of bipolar affective disorder and those having any other neurological symptoms and signs.
\end{abstract}

\section{INTRODUCTION}

Neurocysticercosis is primarily endemic in developing countries and is the most common helminthic infection of the nervous system caused by flatworm Taenia solium, which is normally transmitted between humans and pigs. ${ }^{2}$ Manifestations of neurocysticercosis can be variable depending on the site of the infection. ${ }^{12}$ The unpredictable nature of the immunological reactions of the host against the cysterci as well as the pleomorphic lesions in the central nervous system makes neurocysticercosis an intriguing disease. ${ }^{2}$

\section{CASE REPORT}

A 23-year-old female, married Hindu graduate, resident of Uttar Pradesh, with no family history of mental illness, presented with manic symptoms of overtalkativeness, overcheerfulness, grandiose idea that she is a goddess, ideas of persecution against family members, decreased need for sleep, increased psychomotor activity, muttering to self, followed by two generalised tonic-clonic seizure episodes in April 2018. The patient first had seizures at the age of 13. She had been treated with antiepileptics since then until the age of 20 when she got married in the year 2016. The patient got pregnant in February 2017, and in September 2017, at the 8th month of pregnancy, the patient had a mood of sadness, irritability, crying spells, hopelessness, suicidal ideations and social withdrawal, and she was started on desvenlafaxine $100 \mathrm{mg}$ that was continued until 2 months post partum until January 2018 after complete resolution of the episode.

In April 2018, at the onset of her manic symptoms, she was started on steroids dexamethasone $8 \mathrm{mg}$ and antiepileptics (phenytoin $200 \mathrm{mg}$ ), and she was referred to higher centre for further investigations and treatment.

Her blood tests including red cell count, packed cell volume, erythrocyte sedimentation rate, urea, sugar and liver function test, thyroid profile and electrocardiogram (ECG) were unremarkable exceptfor white cell count, which was raised to $13.1 \times 10^{9} / \mathrm{L}$, suggestive of infection. Serum Venereal Disease Research Laboratory test and stool examination were negative for T. solium. In addition, examination of the fundus did not show any sign of raised intracranial pressure or deposits of cysticerci. X-rays of the skull, cervical spine, chest, forearm and upper arm did not show any calcification. Electroencephalogram was within normal limits. Nerve conduction study showed no sign of peripheral neuropathy. On mental status examination, the patient showed increased psychomotor activity, elated inappropriate affect, increased speech productivity and decreased reaction time with elementary auditory hallucinations, grandiose idea of being a goddess, impaired attention and concentration, impaired immediate and recent memory, impaired judgement and insight. However, abstraction was intact. Psychological testing, that is, Rorschach testing and Bender Gestalt revealed evidence of organic aetiology. 

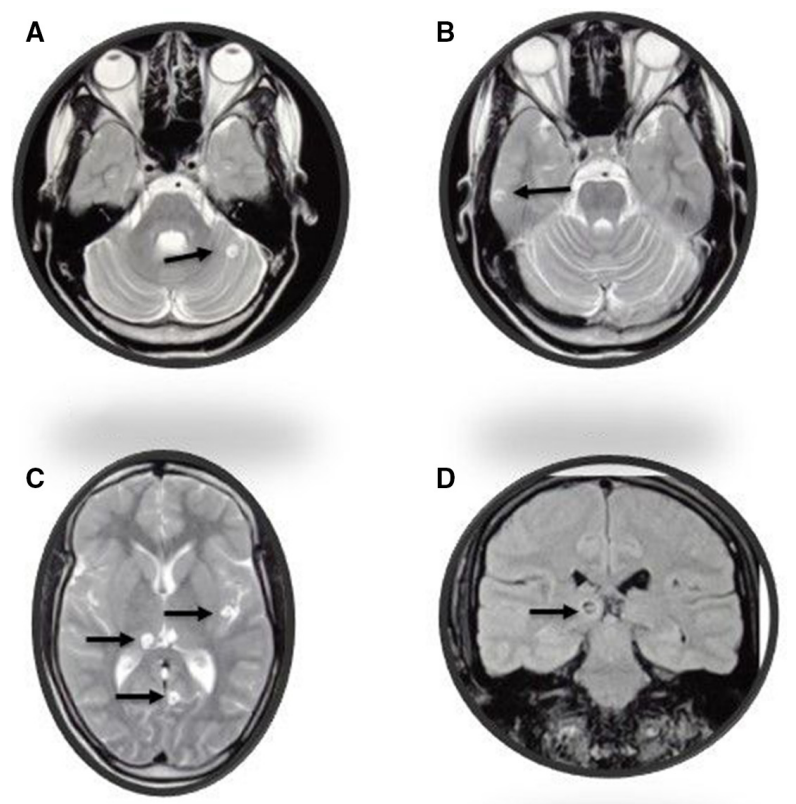

Figure $1 \mathrm{MRI}$ of the brain. (A) T2 weighted image shows a round-to-oval-shaped hyperintense lesion involving the left cerebellar hemisphere. (B) Another similar lesion is seen in the right temporal lobe. (C) Bilateral thalami, left insular cortex and left occipital lobes also show the typical lesions. (D) Fluid-attenuated inversion recovery image shows hypointense lesion with eccentric nodule and surrounding hyperintense signal suggestive of perilesional oedema. MRI, magnetic resonance imaging.

The patient was advised for a magnetic resonance imaging (MRI) of the brain in April 2018 (figure 1). The MRI showed multiple T2 hyperintense round-tooval-shaped lesions with surrounding hyperintense signals on fluid-attenuated inversion recovery images in the bilateral cerebral hemisphere and cerebellar hemisphere as depicted in figure 1 . Some of the lesions were showing eccentric nodules. No post-contrast enhancement was seen suggestive of the colloidal vesicular stage of neurocysticercosis.

In July 2018, when manic symptoms were resolved, computed tomography (CT) scan showed multiple diffusely scattered tiny calcified lesions in the bilateral cerebral hemisphere as depicted in figure 2. Similar lesions were also present in occipital lobes and external capsules (not shown). No appreciable perilesional oedema was seen on non-contrast CT suggestive of the calcified nodular stage of neurocysticercosis. Another MRI in 2020 was done to look for the resolution of the disease, which showed multiple lesions as calcified (including the right temporal lobe lesion) and some of them were showing surrounding perilesional oedema but no enhancement suggestive of granular nodular stage and some of them were in the colloidal vesicular stage, which were showing enhancement and perilesional edema as depicted in figure 3 .
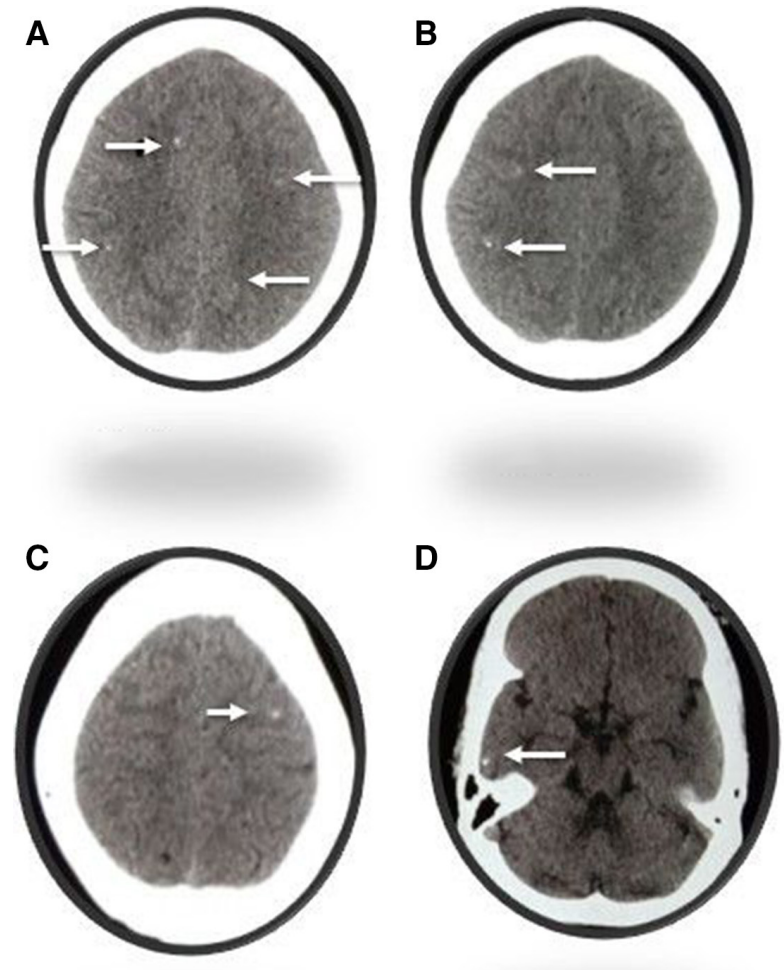

Figure 2 CT of the brain. (A) Multiple tiny intra-axial hyperdense foci (white solid arrow) are seen in the frontal and parietal lobes on both sides. (B) Two hyperdense foci are seen in the right frontal and parietal lobes. (C) Tiny hyperdense focus in the left frontal lobe. (D) Another hyperdense focus in the right temporal lobe. CT, computed tomography.

Hence, the possibilities of tuberculoma, neurosyphilis and space-occupying lesion in the brain were excluded by imaging and blood tests.

At the higher centre, considering her symptoms, the patient was started on risperidone built up to $6 \mathrm{mg}$ for the management of psychotic symptoms, and for epilepsy, tablet carbamazepine was started to use its additional action as a mood stabiliser. The patient showed improvement within 4 months and it was planned to give her steroids along with antihelmintics. The patient continued taking antiepileptics carbamazepine $600 \mathrm{mg}$ and risperidone tapered down and stopped slowly over 2 years, and most of the lesions seemed to have calcified in subsequent radiological scans. The patient still had occasional headaches and dysmorphopsia probably owing to perilesional oedema in a few of the lesions and was started on dexamethasone $10 \mathrm{mg}$ and albendazole $1200 \mathrm{mg}$. The patient reported improvement in these symptoms. She has been advised to continue the treatment and regular follow-up. In addition, it is planned to do a detailed neurological examination to find out any sign suggestive of reactivation of lesions in every follow-up visit and radiological investigations if required.

After 2 years of follow-up, in August 2020, the patient's radiological findings were multiple lesions in bilateral 
A
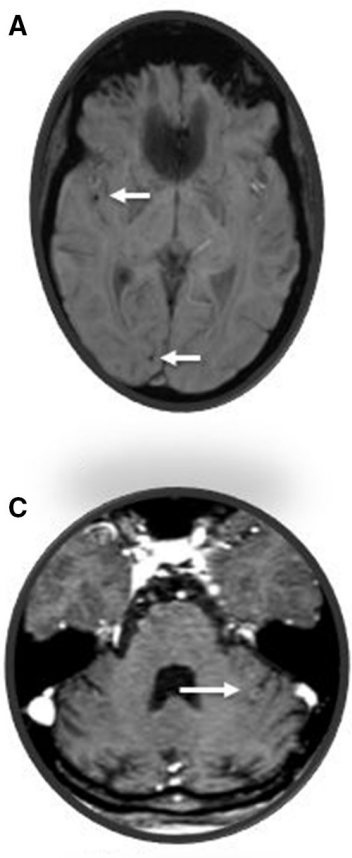

B
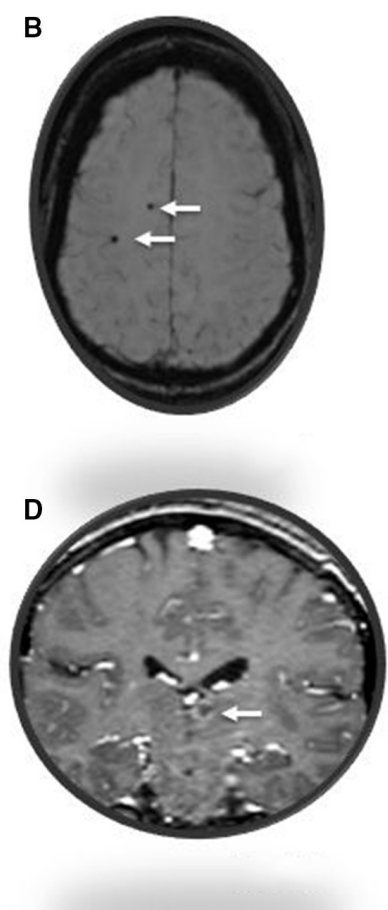

Figure $3 \mathrm{MRI}$ of the brain. (A,B) Tiny susceptibility artefacts as hypointense foci on susceptibility-weighted imaging suggestive of calcifications in the temporal lobe, occipital lobe, frontal and parietal lobes in the right cerebral hemisphere. (C) T1 weighted fat-saturated post-contrast image shows a faintly ring-enhancing lesion in the left cerebellar hemisphere. (D) T1 weighted fat-saturated postcontrast image shows another ring-enhancing lesion in the left thalamus. MRI, magnetic resonance imaging.

cerebral lobes, basal ganglia and left cerebellar parenchyma, a few of which are showing eccentric scolex and a few of which are calcified with mild perilesional oedema suggestive of multiple neurocysticerci in different stages of development. The current mental status examination of the patient was not suggestive of any other cognitive impairment except difficulty in sustaining attention. Figure 4 shows the chronology of events in summary.

\section{DISCUSSION}

Pregnancy and the postpartum period make women vulnerable to psychiatric disorders owing to hormonal turmoil. In addition, pregnancy is an immune-variable state and makes women vulnerable to certain infections. So, hormonal turmoil and immune system variability during pregnancy might have allowed the neurocysticercosis to flare up and caused reactivation of neurocysticercosis, which might have led to depressive symptoms at the beginning of the inflammatory process. In addition, the evidence is suggestive of reactivation of lesions of neurocysticercosis in this period owing to immune system variability of pregnancy. ${ }^{3-5}$

These depressive symptoms were considered as commonly experienced depressive disorder and managed accordingly with desvenlafaxine by the local practitioner.

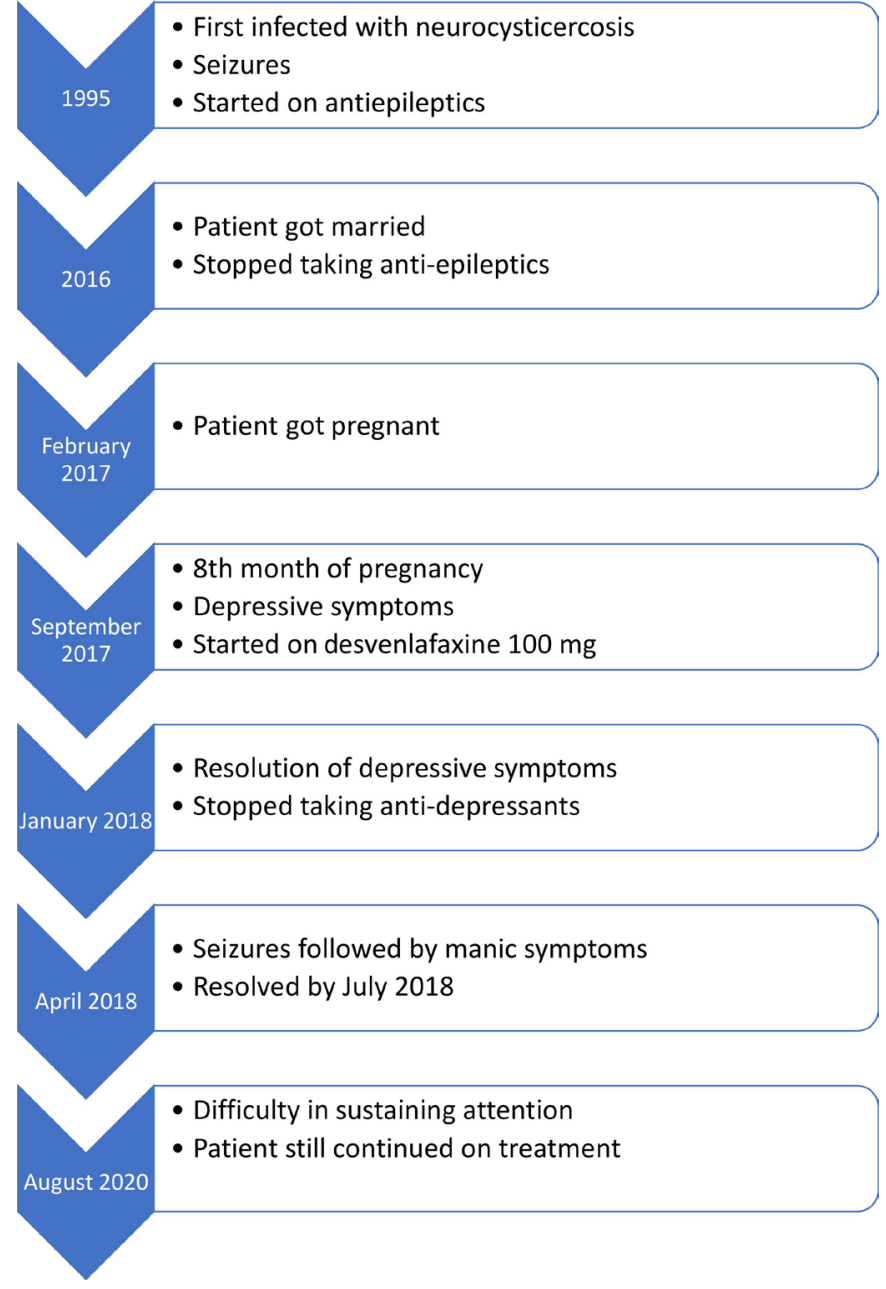

Figure 4 Timeline of the key events.

The flare of neurocysticercosis might have led to depression in the late pregnancy and postpartum period but since this period is commonly associated with peripartum and postpartum depression, it might have been considered as independent depression and managed accordingly with desvenlafaxine by the local practitioner.

During the postpartum period, the patient developed seizures and manic symptoms, which made the practitioner refer the patient to a higher centre for better management. The seizure episodes created the suspicion of having an underlying neurological illness that might be the reason for bipolar affective disorder. So, a detailed neurological evaluation was done to find out the reason for epilepsy. The flared lesions of neurocysticercosis were found with perilesional oedema involving the right temporal lobe lesion, which is suggestive of manic symptoms.

Seizures are the most common manifestation of neurocysticercosis occurring in about $70 \%-90 \%$ of patients ${ }^{1}$ and only about $5 \%$ of patients with neurocysticercosis present with neuropsychiatric manifestations. ${ }^{1}$ Forlenza et al studied psychiatric morbidities in 38 patients with neurocysticercosis and found that depression was the most frequent psychiatric diagnosis (52.6\%), 14.2\% of 
patients reported psychotic symptoms and only $2.6 \%$ showed manic symptoms as presenting symptoms. ${ }^{6}$ The degenerating cysts and reactive inflammation within the adjacent nervous tissue might be a strong determinant of neurocysticercosis-induced epilepsy and may also trigger psychiatric symptoms depending on the location of cysts and vulnerability of the individual. ${ }^{7}$

Frontal lobe damage may lead to mania-like symptoms, disinhibition, irritability, impaired judgement and personality changes. ${ }^{8}$

Lesions in the right temporal lobe are often associated with manic presentation as seen in our case. The prevalence of mania in neurocysticercosis is $2.6 \%$ leading to rare presentation.

Our case is a rare presentation as it typically started with a classic depressive episode, and then manic symptoms and seizure episodes were the only reasons to suspect an underlying neurological illness. In addition, detailed history taking can make a difference as the patient was already diagnosed with epilepsy and the reason for epilepsy was not ruled out during the initial presentation of depressive symptoms as it might have been assumed to be idiopathic epilepsy.

So, keeping the worldwide prevalence of neurocysticercosis in mind, it is important to rule it out in all patients with neuropsychiatric symptoms and seizures, as appropriate treatment can save the life of an individual.

Acknowledgements Dr Nitin Kumar, Senior Resident, Department of Psychiatry, VMMC \& Safdarjung Hospital, New Delhi, for helping in the collection of relevant articles. Dr KS Anand and Dr Jyoti Garg, Professor, Department of Neurology, ABVIMS \& Dr RML Hospital, New Delhi, for discussing the neurological aspects of the case and helping in clinical management of the patient.

Contributors The authors confirm contribution to the paper as follows: study conception and design-SB and LSS; data collection-SB and SK; analysis and interpretation of results—SB, SK and LSS; draft manuscript preparation-SB, SK and LSS. All authors reviewed the results and approved the final version of the manuscript.

Funding The authors have not declared a specific grant for this research from any funding agency in the public, commercial or not-for-profit sectors.

Competing interests None declared.

Patient consent for publication Obtained.

Ethics approval This study involves human participants but Post Graduate Institute of Medical Education and Research, Dr RML Hospital, New Delhi, Institutional Ethics Committee exempted this article as this is a case report of a single patient and requires patient consent only. Participants gave informed consent to participate in the study before taking part.

Provenance and peer review Not commissioned; externally peer reviewed.

Open access This is an open access article distributed in accordance with the Creative Commons Attribution Non Commercial (CC BY-NC 4.0) license, which permits others to distribute, remix, adapt, build upon this work non-commercially, and license their derivative works on different terms, provided the original work is properly cited, appropriate credit is given, any changes made indicated, and the use is non-commercial. See: http://creativecommons.org/licenses/by-nc/4.0/.

ORCID iD

Surbhi Batra http://orcid.org/0000-0003-1019-7831

\section{REFERENCES}

1 Carabin $\mathrm{H}$, Ndimubanzi PC, Budke CM, et al. Clinical manifestations associated with neurocysticercosis: a systematic review. PLoS Negl Trop Dis 2011;5:e1152.

2 Del Brutto OH. Neurocysticercosis: a review. ScientificWorldJournal 2012;2012:1-8.

3 D'Cruz RF, Ng SM, Dassan P. Neurocysticercosis in pregnancy: maternal and fetal outcomes. Oxf Med Case Reports 2016;2016:138-40.

4 Garweg JG, Scherrer J, Wallon M, et al. Reactivation of ocular toxoplasmosis during pregnancy. BJOG 2005;112:241-2.

5 Webb C, Rosa ML, Olson G, et al. Neurocysticercosis in pregnancy. AJP Rep 2018;8:e51-6.

6 Forlenza OV, Filho AH, Nobrega JP, et al. Psychiatric manifestations of neurocysticercosis: a study of 38 patients from a neurology clinic in Brazil. J Neurol Neurosurg Psychiatry 1997;62:612-6.

7 Mahajan SK, Machhan PC, Sood BR, et al. Neurocysticercosis presenting with psychosis. J Assoc Physicians India 2004;52:663-5.

8 Mishra BN, Swain SP. Psychiatric morbidity following neurocysticercosis. Indian J Psychiatry 2004;46:267-8.

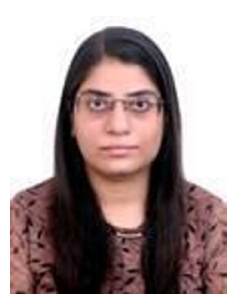

Dr Surbhi Batra has done her M.B.B.S. from VMMC and Safdarjung Hospital, New Delhi, and postgraduate training (M.D. Psychiatry) from ABVIMS and Dr. Ram Manohar Lohia Hospital, Centre of Excellence in Mental Health, New Delhi (India), and passed out in July 2021. At present, she is working at ABVIMS and Dr. Ram Manohar Lohia Hospital, New Delhi (India), as a senior resident in Psychiatry. She served as a General Secretary of Student Wing and a Member of Central India Unit UNESCO Chair in Bioethics, Haifa, for the academic year 2019-2020. She has also received the award for "Most Innovative Team" in the event "Ethiscan" in the National Bioethics, Medical and Research Conference, ETHOS 2018. She has presented multiple papers and posters, both at national and international conferences. Her main research interests include Adult Psychiatry, Obsessive-Compulsive Disorder, Bioethics and Forensic Psychiatry. 